Electromechanical Wave Imaging of Normal and Ischemic Hearts

\title{
In Vivo
}

\author{
Jean Provost, \\ Department of Biomedical Engineering, Columbia University, New York, NY 10027 USA \\ Wei-Ning Lee, \\ Department of Biomedical Engineering, Columbia University, New York, NY 10027 USA \\ Kana Fujikura, and \\ Department of Biomedical Engineering, Columbia University, New York, NY 10027 USA \\ Elisa E. Konofagou \\ Departments of Biomedical Engineering and Radiology, Columbia University, New York, NY \\ 10027 USA
}

Elisa E. Konofagou: ek2191@columbia.edu

\section{Abstract}

Electromechanical wave imaging (EWI) has recently been introduced as a noninvasive, ultrasound-based imaging modality, which could map the electrical activation of the heart in various echocardiographic planes in mice, dogs, and humans in vivo. By acquiring radiofrequency (RF) frames at very high frame rates $(390-520 \mathrm{~Hz})$, the onset of small, localized, transient deformations resulting from the electrical activation of the heart, i.e., generating the electromechanical wave (EMW), can be mapped. The correlation between the EMW and the electrical activation speed and pacing scheme has previously been reported. In this study, we pursue the development of EWI and analysis of the EMW properties in dogs in vivo for early detection of ischemia. EWI was performed in normal and ischemic open-chested dogs during sinus rhythm. Ischemia of increasing severity was obtained by gradually occluding the leftanterior descending (LAD) coronary artery. We also introduce the novel method of motionmatching that achieves the reconstruction of the full EWI ciné-loop at very high frame rates even when the ECG may be irregular or unavailable. Incremental displacements were previously used by our group to map the EMW. This paper focuses on the associated incremental strains, which facilitate the interpretation of the EMW by relating it directly to contraction. Moreover, we define the onset of the EMW as the time, at which the incremental strains change sign after the onset of the QRS complex of the ECG. Based on this, isochronal representations of the EMW were generated using a semi-automated method. The isochronal representation of the EMW during sinus rhythm was reproducible and shown similar to electrical activation maps previously reported in the literature. After segmentation using a contour-tracking method, the two- and four-chamber views were imaged and displayed in bi-plane views, allowing a 3-D interpretation of the EMW. EWI was shown to be sensitive to the presence of intermediate ischemia. EWI localized the ischemic region when the LAD was occluded at $60 \%$ and beyond and was capable of mapping the increase of the ischemic region size as the LAD occlusion level increased. In conclusion, the activation maps and wave patterns obtained with EWI were similar to the electrical equivalents previously reported in the literature. Moreover, EWI was found to be sensitive enough to detect and map intermediate ischemia. Those results indicate that EWI could be used to assess electrical 
conduction properties of the myocardium, and detect ischemic onset and disease progression entirely noninvasively.

\section{Index Terms}

Electrical mapping; electromechanical wave imaging; high framerate echocardiography; ischemia; radio-frequency speckle-tracking

\section{Introduction}

Electrical mapping of the heart has emerged as an important tool for treatment monitoring of arrhythmias such as ventricular tachycardia. Mapping of local timings of electrical activation in the ventricle can identify abnormally conducting regions and guide radiofrequency (RF)-ablation treatments. Currently available clinical mapping systems are, however, invasive, since they require catheterization for introduction into the heart chamber. Therefore, electrical mapping cannot be performed for early detection of diseases or followup of chronic diseases such as heart failure. Electromechanical wave imaging (EWI) [1]-[3] has recently been introduced as a non-invasive, noncontact, ultrasound-based modality, which could map the transmural electrical activation of the heart within various echocardiographic planes. This imaging modality is based on the measurement of small, transient deformations occurring in the myocardium a few milliseconds after (equal to the electromechanical delay), but following similar propagation patterns as, the electrical activation. More specifically, those deformations are the result of the cardiac excitationcontraction coupling: once the action potential reaches the myocytes, the latter undergo depolarization followed by an uptake of calcium, which triggers contraction a few milliseconds later [4]. Therefore, by measuring the onset of this contraction, the activation pattern across the entire myocardium can be mapped.

Over the past two decades, several methods have been developed for measuring deformations using ultrasound-based methods. Two-dimensional speckle-tracking-based motion estimation techniques have been implemented in clinical systems. Different approaches based on B-Mode [5] or radio-frequency (RF) speckle tracking [6]-[9] or phasetracking techniques [10] have also been proposed in the literature for myocardial contractility assessment. Recently, open architecture ultrasound systems enabled motion estimation at very high effective RF-frame rates [11] of standard echocardiographic views. The full view of the heart is divided into five to seven sectors acquired at very high frame rates and a full-view ciné-loop is then reconstructed via ECG gating. Such high frame rates and the RF phase information increase the estimation quality and thus the reliability of 2-D displacement and strain mapping [8].

The increase in the frame rate did not only allow for better precision of the RF-based motion estimation, but also achieved a temporal resolution at the same time scale as that of the electrical propagation. More specifically, it allowed the detection of transient phenomena that occur during both isovolumic phases [11]. For example, it has been shown possible to identify the mechanical waves in the myocardial wall occurring when the valves open and close [1], [2], [12]. Propagating incremental displacement generated by the early contraction of myocytes, i.e., the electromechanical wave (EMW), has been depicted on EWI ciné-loops and images, and its correlation with the electrical activation velocity [1], [2] and pacing scheme [3] has been verified. More recently, the EMW was reproduced in simulations and shown to be correlated with simulated and experimental electrical activation patterns [13]. 
Alternative methods for assessment of local electrical properties in vivo involve the use of electrode arrays [14], either by mounting an electrode sock around the heart through openheart surgery [15], [16] to map the epicardial activation or, using electrode catheters [17], [18]. Newly developed noninvasive techniques based on surface potentials provided fully 3D activation sequences [19]. A method based on magnetic resonance (MR) tagging has also been proposed [20], where the subepicardial contraction sequence was mapped and compared to the electrical activation maps obtained with an epicardial electrode sock.

In this paper, we pursue the development of EWI and the analysis of the EMW properties in dogs in vivo. In previous reports [11], high frame rates were obtained using ECG gating. However, for the analysis of diseases such as ventricular tachycardia, the ECG may not be regular. For example, when atrio-ventricular dissociation occurs, the atria and ventricles follow different rhythms, which may compromise the use of ECG for co-registration of adjacent sectors. In the first part of this paper, we present the new technique of motionmatching that is based on local incremental displacements used to co-register those sectors. Incremental displacements were previously used to map the EMW [2], [3]. While this approach provides a higher signal-to-noise ratio, it may be affected by the presence of rigid motion. This paper focuses on the associated incremental strains, which facilitate the interpretation of the EMW by relating it directly to contraction. More precisely, we define the onset of the EMW as the time, at which the cardiac muscle transitions from thinning to thickening or vice versa. The EMW was imaged during sinus rhythm in five normal canine hearts before and after graded ischemia in vivo. We show the reproducibility of EWI during sinus rhythm and compare the propagation patterns of the EMW to those of the electrical propagation reported in the literature. A preliminary feasibility study on the capability of EWI to detect degrees of ischemia was conducted in a single case. Graded ischemia was obtained by gradually occluding the left-ascending coronary artery to generate an ischemic region of increasing size and severity, in order to test the EWI performance in depicting the ischemic region size as the coronary flow decreases.

\section{Methods}

\section{A. Experimental Protocol}

In this study, approved by the Institutional Animal Care and Use Committee of Columbia University, five mongrel dogs of either sex, ranging from 23 to $32 \mathrm{~kg}$ in weight, were anesthetized with an intravenous injection of thiopental $(10-17 \mathrm{mg} / \mathrm{kg})$. They were mechanically ventilated with a rate- and volume-regulated ventilator on a mixture of oxygen and titrated isoflurane $(0.5 \%-5.0 \%)$. Morphine $(0.15 \mathrm{mg} / \mathrm{kg}$, epidural $)$ was administered before surgery, and lidocaine ( $50 \mathrm{micrograms} / \mathrm{kg} / \mathrm{h}$, intravenous) was used during the entire procedure. To maintain blood volume, $0.9 \%$ saline solution was administered intravenously at $5 \mathrm{~mL} / \mathrm{kg} / \mathrm{h}$. The animal was positioned supine on a heating pad.

Standard limb leads were placed for surface electrocardiogram (ECG) monitoring. A solid state pressure transducer catheter (Millar Instruments, Houston, TX) was inserted into the left-ventricular cavity via the right carotid artery, the aortic root, and across the aortic valve. Oxygen saturation of the blood and peripheral blood pressure were monitored throughout the experiment. The chest was opened by lateral thoracotomy using electrocautery. After removal of the pericardium, a customized constrictor and a flow probe (Transonic Systems, Inc.) were positioned immediately distal to the first diagonal of the left anterior descending (LAD) coronary artery to induce graded LAD occlusion and thus variable ischemic levels at $20 \%$ decrements of the initial coronary blood flow.

A total of 12 piezoelectric crystals (Sonometrics Corporation, London, ON, Canada) were then implanted in the left ventricular wall. For endocardial and mid-wall crystals, an $18 \mathrm{G}$ 
needle was used for insertion. All crystals were maintained in position after placement using silk sutures. Echocardiography was performed at each LAD occlusion level. Forty-five minutes after complete LAD occlusion, the constrictor was released for complete reperfusion and the same scanning procedure repeated. The heart was then excised and sectioned in $1 \mathrm{~cm}$ transverse slices to perform pathology. The sliced heart sections were immersed in a $1 \%$ Triphenyltetrazolium chloride (TTC) solution and incubated at $37{ }^{\circ} \mathrm{C}$ for $1.5 \mathrm{~h}$. The sections were finally fixed in a $10 \%$ formalin solution for $30 \mathrm{~min}$. A pale region indicated the site of the ischemia/infarction, while the red color represented the viable cardiac muscle.

\section{B. Echocardiography}

An Ultrasonix RP system with a 3.3 MHz phased array was used to acquire RF frames from 390 to $520 \mathrm{frames} / \mathrm{s}$ (fps) using a customized, automated composite technique [11]. Briefly, to increase the resulting frame rate, the image was divided into partially overlapping sectors corresponding to separate cardiac cycles. A full-view image was then reconstructed by using the motion-matching technique (see Section II-B2). To minimize motion artifacts, the probe was attached to a stabilizer (Medtronic Corporation, Minneapolis, MN) and the respirator was interrupted for 6-20 s depending on the acquisition. The cavity was filled with degassed ultrasound gel to ensure acoustic coupling and to avoid contact between the heart and the probe. Two views were considered in this paper, i.e., an oblique parasternal four-chamber view [Fig. 1(a)] and a parasternal two-chamber view [Fig. 1(b)].

1) Incremental Displacement and Strain Estimation-Axial (parallel to the ultrasound beam) incremental displacements were estimated offline using an RF-based cross-correlation method (window size: $4.6 \mathrm{~mm}, 80 \%$ overlap). Axial incremental strains were then obtained using a least-squares estimator [21] with a kernel equal to $6.75 \mathrm{~mm}$. In this study, we considered incremental strains, i.e., the local change in length was measured with respect to the previous frame, using the Eulerian description [22], i.e., the material points were not followed. Since the displacements occurring during the QRS complex were small, the temporal incremental strain curves obtained in Eulerian and Lagrangian descriptions were expected to be similar. We chose to use the Eulerian description to avoid cumulative numerical errors associated with calculations of the incremental strains when using a Lagrangian description. The myocardium was segmented using an automated contour tracking technique [23], and displacement and strain maps were then overlaid onto the B-mode images. Two views were temporally coregistered using the ECG signals and spatially coregistered by an echocardiography expert and displayed in a 3-D biplane view in Amira 4.1 (Visage Imaging, Chelmsford, MA).

\section{2) Automatic, Composite, High Frame-Rate, Full View Imaging Using Motion} Matching-The motion-matching algorithm is illustrated in Fig. 2. Five to seven sectors of 12 to $16 \mathrm{RF}$ beams each were acquired consecutively at up to 520 frames per second (fps). Fig. 2(a) shows an example using two of those sectors. Three RF beams (in purple) on both sides of each sector overlapped for a full-view image containing 65 beams. Acquisition of each sector did not start at the same time-point in the heart cycle. Therefore, to reconstruct a full-view ciné-loop, it was necessary to identify, and correct for, the temporal offset between sectors. A conventional approach to solving this problem would be to acquire ECG signals along with each sector. The temporal offset between sectors could then be estimated by the offsets between the R-peaks of the ECG signals corresponding to each sector. As the method is also aimed for detection of conduction abnormalities, it is essential that the technique used here does not depend on the reproducibility of the ECG. For example, during ventricular tachycardia, the ventricles and the atria may follow two distinct rhythms. The ECG will thus encompass both rhythms and the P-and R-waves may coincidentally have the same time 
occurrence, occasionally generating a fused waveform. An ECG-based method in that case might be difficult to implement, although the mechanics of the ventricles may still be periodic. The alternative proposed here is to use the temporal variation of the incremental displacements measured in the overlapping RF beams. Therefore, the motion-matching approach utilizes the local periodicity of the heart motion, rather than its global conduction properties. This also simplifies the instrumentation by not requiring simultaneous ECG acquisition. More precisely, if we assume the heart undergoes periodic motion, the same information is acquired twice in the overlapping regions. The periodicity of the heart implies that the following equation holds for all sectors $k$, depths $r$, angles $\theta$, and times $t$

$$
D_{k}(r, \theta, t)=D_{k}\left(r, \theta, t+n_{k} T\right)
$$

where $D$ denotes the displacement estimate, $T$ denotes one heart cycle duration and $n_{k}$ is an integer. Each sector is delayed with respect to the preceding by an unknown amount of time. This delay can then be written as $n_{k} T+t_{k}$, with $t_{k}<T$. For the overlapping RF beams, denoted by a prime, $\theta^{\prime}$, the following equation holds:

$$
\begin{gathered}
D_{k+1}\left(r, \theta^{\prime}, t\right)=D_{k}\left(r, \theta^{\prime}, t+n_{k} T+t_{k}\right) \\
=D_{k}\left(r, \theta^{\prime}, t+t_{k}\right)
\end{gathered}
$$

Reconstructing the full-view image is equivalent to estimating $t_{k}$ for all $k$. This is achieved by finding the time associated with the peak of the cross-correlation function

$$
t_{k}=\arg \max _{r, t^{\prime}} \int D_{k+1}\left(r, \theta^{\prime}, t\right) D_{k}\left(r, \theta^{\prime}, t+t^{\prime}\right) d t .
$$

This approach is illustrated in Fig. 2(a). Equation (3) was implemented numerically and the true peak was approximated using cosine interpolation [24]. One of the main advantages of the motion-matching technique is that the cross-correlation method also provided a correlation coefficient that indicated the quality of the sector matching. A high correlation coefficient will ensure continuity of the reconstructed B-mode, and of the reconstructed displacement and strain maps and cine-loops across the sectors. As the experiment conducted in this study usually comprises two to three heart cycles per sector, it was then possible to select the best combination out of multiple heart cycles. For example, if two heartbeats were acquired per sector over seven sectors, it would be possible to choose among 128 combinations of heart cycles. Fig. 2(b) indicates good matching between the temporal profile of incremental displacements obtained in overlapping RF beams during two different heartbeats in a normal, open-chest, canine heart. Fig. 2(c) displays the temporal correlation coefficient along the same RF beam. A correlation coefficient close to 1 is obtained at the level of the myocardium, while the correlation was low in the blood cavities (due to blood flow and low scattering) and in the surrounding ultrasound gel (which does not undergo periodic motion).

\section{Electromechanical Wave Mapping}

Fig. 3 shows typical temporal incremental strain profiles over time in three locations in the septum where the axial direction matches the cardiac radial coordinate. Before the first deflection of the QRS complex, the cardiac muscle is in a thinning state. A few milliseconds later, i.e., after the electrical activation of the ventricles is initiated, a regional transition from thinning to thickening can be observed as a transition from negative to positive 
incremental strains. Similar incremental strain profiles are observed in both the two- and four-chamber views. However, in regions such as the apex, where the axial direction closely aligns with the cardiac longitudinal one, a transition from positive to negative incremental strains is observed. In the general case, the axial direction contains the projection of the motion in the cardiac geometry coordinates, and is thus a linear combination of the radial, longitudinal and shear directions. The EMW is therefore defined as the time-point, at which the incremental axial strains change sign or, zero-cross, immediately following the onset of the $Q$-wave. To visualize the EMW wavefront, the incremental strains were then saturated symmetrically around zero. Consequently, the zero-crossing point appeared as a sharp transition between blue and red maps in the full 2-D views [Figs. 4(b) and 5]. In the case of the views considered in this study, axial strains will contain, in most regions, a large projection of the radial component. Therefore, contraction and, hence, activation, will result mostly in myocardial tissue thickening. Regions where the axial direction does not contain a large projection of the radial component must be interpreted with caution, as the axial incremental strains, in this case, contain information about both the radial and longitudinal strains. Those regions include the apical [Fig. 4(b)] and basal regions of the anterior and lateral wall, in the two- and four-chamber view, respectively. To generate an isochronal representation of the EMW, a semi-automated method was developed. Regions of the myocardium were first manually selected. For each of those regions, a time interval containing the zero-crossing point of the incremental strains was manually selected. The time of occurrence of the zero-crossing point was then obtained by minimizing the smoothing spline approximation of the absolute value of the incremental strains within this interval. As a result, the time of arrival of the EMW was identified as it propagated in multiple regions of the myocardium. A full map of arrival times was then generated via bicubic interpolation. When the waveform was not sufficiently clear to identify the zerocrossing point in a region because the activation occurred after the onset of the isovolumic contraction phase, or because of noise, this region was omitted in the isochronal representation and displayed in black.

\section{Results}

\section{A. EWI of the Normal Heart}

Fig. 4(a) shows the propagation of incremental displacement in the four-chamber view: red indicates movement upwards and blue indicates movement downwards. Therefore, in that view, a contracting heart is mapped in blue at the septal and right-ventricular walls and in red at the lateral wall. Fig. 4(a) shows the corresponding displacement patterns propagating from base to apex in the septal and lateral walls, and propagation of downward motion in the right-ventricular wall.

A least-squares strain estimator [21] was then applied on the incremental displacement data, providing the incremental strain information [Fig. 4(b)]. Red indicates regional thickening while blue indicates regional thinning. In regions, where the axial direction coincides mainly with the radial direction, contraction is mapped as thickening of the myocardium. More specifically, two activation sites at the mid-basal level were visible approximately $30 \mathrm{~ms}$ after the $Q$-wave in both the left endocardium of the septum and in the endocardium of the lateral wall. This is a thickening (red) wave that travels towards both the base and the apex. At approximately $40 \mathrm{~ms}$ after the onset of the QRS complex, a thickening wave becomes visible in the right-ventricular wall, which travels principally towards the base. Noticeably, $60 \mathrm{~ms}$ after the onset of the $Q$-wave, the basal parts of the three walls are still thinning. Note that in the apical region where the axial direction coincides with the longitudinal direction of the cardiac geometry, the myocardium is depicted undergoing longitudinal shortening rather than radial thickening due to the aforementioned angle dependence. 
Fig. 5 shows the electromechanical wave in the parasternal two-chamber view. In the posterior wall, it was possible to observe a wave traveling from the mid-basal level to the apex and later to the base.

Fig. 6 shows the isochrones associated with the EMW propagation in two different dogs. A similar propagation was observed in all five dogs: in the four-chamber view [Figs. 4(b) and 6], the electromechanical wave was initiated at the mid-cavity segment (see [25] for a description of the standardized myocardial segmentation) in the septal and in the lateral walls, traveling towards the apex and base. In the right-ventricular wall, the EMW appeared on the endocardium near the apex a few milliseconds later, and traveled towards the base. In the two-chamber view (Figs. 5 and 6), the wave was initiated in the mid-cavity segment in the posterior wall and closer to the apex in the anterior wall.

\section{B. EWI of the Ischemic Heart}

Fig. 7 shows the EMW propagation at different levels of ischemia. The time, at which the images are displayed, corresponds to the time, at which the activated region covered the largest portion of the myocardium, i.e., immediately after electrical activation and preceding the closing of the mitral valve. When the LAD was occluded at $60 \%$ and beyond, the ischemic region was easily identified as the region, through which the EMW cannot propagate. The ischemic region appears to grow with the LAD occlusion level until it reaches a maximum size at 100\% LAD occlusion [Fig. 7(f)]. After reperfusion, the size of the ischemic region in the posterior wall decreases.

The presence of acute ischemia was confirmed with pathology, after reperfusion [Fig. 7(i)]. TTC was used to stain nonviable tissue, which generally spans a smaller region than the ischemic one [26]. Reperfusion accentuates the size of this nonviable region [27]. The transverse slice shown in Fig. 7(i) was obtained approximately at $3 \mathrm{~cm}$ from the apex. Fig. 8 shows the comparison between the normal, 60\% LAD occlusion, and 100\% LAD occlusion in a bi-plane view. In the ischemic cases, the wave was initiated as in the normal case, but its propagation was impeded at the apical segment. After EMW propagation, a region that did not undergo thickening could be identified, which indicated an inability of the tissue to contract. The location of the ischemic region is consistent with the pathology findings as shown in Fig. 7(i).

\section{Discussion}

Five canine hearts were imaged before and after ischemia at very high frame rates during sinus rhythm in vivo. The very high frame rates were achieved by using a novel motionmatching technique, which may not require a regular cardiac cycle or electrical conduction pattern to reconstruct a full view ciné-loop. EWI was shown sensitive to the presence of an ischemic region of various sizes. The location of the ischemic zone obtained with EWI was compared with pathology. EWI could thus constitute a completely noninvasive, novel approach to electrical mapping.

\section{A. Normal Heart}

In order to compare the EMW and the electrical activation patterns in the sinus rhythm case, we refer to the intramural electrical activation pathways previously described [28]-[30] for mammalian ventricles. According to [28], the earliest activity in dogs occurs at the region of the terminations of the left bundle on the mid-left septal endocardium at the mid-basal level, a few milliseconds before the QRS deflection. Activity on the right side of the septum occurs slightly later, again at the Purkinje terminals. During the first quarter of the QRS, most of the endocardial layer of the apical and mid-cavity region of the myocardium is depolarized. The activity then propagates from the endocardium to the epicardium, and 
towards both the apex and base. At the R-peak, only portions of the basal and lateral left ventricle and of the basal septum remain to be activated. Other studies performed later in swine, rats, and humans [30], identified three points of early activation: 1) an area high on the anterior paraseptal wall immediately below the mitral valve, 2) an area at half the distance from apex to base in the left side of the interventricular septum, and 3) the posterior paraseptal area at approximately one third of the distance from apex to base.

Those observations are in agreement with results obtained with EWI, when considering that a delay of a few milliseconds (20-40 $\mathrm{ms}$ in vivo [31]) exists between the electrical and mechanical activation of the myocardium, namely the electromechanical delay. In the five canine hearts imaged in this study, the EMW was initiated in the mid-cavity segment on the left side of the septum, and from the endocardium of the lateral wall near the base. It was also initiated from the posterior and anterior mid-cavity segments in the two-chamber view. In the right-ventricular wall, the wave was initiated approximately $19 \mathrm{~ms}$ later and traveled towards the base. Propagation also occurred trans-murally, i.e., from the endocardium to the epicardium. Differences with the literature in the two-chamber view could be explained by the choice of the plane that may not coincide with the regions cited in [30], along with the fact that those studies were conducted on different species. Moreover, basal wall regions remained inactivated even $60 \mathrm{~ms}$ after the onset of the $Q$-wave. This is also in agreement with previous reports [28] indicating that at the R-peak, basal regions are not electrically activated yet. Considering the electromechanical delay, it is then expected that the basal region will not contract before the onset of the iso-volumic contraction phase occurs, in the vicinity of the R-peak, where the mechanical waves induced by the mitral valve closure do not allow a clear identification of the EMW onset. The isochrones obtained in each heart showed similar propagation patterns. Two different cases are shown in Fig. 6. Although differences exist between the two cases, the origins of the activation were similar, on the lateral, septal, and right-ventricular walls, as well as in the anterior and posterior walls. The reproducibility of these observations is an indicator that the normal heart has a specific EMW propagation pattern, and that a modification to this pattern could originate from an electrical abnormality.

The EMW propagation is also disrupted (Fig. 4) after the mitral valve closes a few milliseconds after the R-peak, approximately at $45 \mathrm{~ms}$ after the onset of the QRS complex. At that time, oscillations become visible in the temporal incremental strain profiles (Fig. 3; yellow circle). Although this phenomenon has already been documented [2], [12], [32]-[34], the techniques developed here for EWI could constitute an interesting tool for its visualization and quantification. This phenomenon may, however, occur prior to the complete mechanical activation of the entire ventricle, thereby also reducing the quality of the correlation between the EMW and the electrical activation pattern. Simulations [13] or appropriate modeling may provide a correction for these sources of error in the future.

\section{B. Ischemic Heart}

The presence of ischemia impeded the EMW from propagating. This is expected, since during the first few minutes of ischemia, the myocardium gradually loses its ability to generate systolic force [35]. In other words, even if the electrical activation did occur, the ischemic region would not contract in response to it. Previous studies [36] confirmed that 5 min of experimental ischemia in dogs converted the normal pattern of systolic circumferential and longitudinal shortening and radial thickening to circumferential and longitudinal thickening and radial shortening. Earlier studies [37] found the relationship between regional myocardial shortening and the level of blood flow irrigating that region to be exponential, i.e., that the decrease in regional thickening becomes more important as the coronary flow is diminished, and that a $20 \%$ LAD occlusion level was sufficient to significantly impair cardiac function. 
In the present study, an ischemic region could be clearly identified when the LAD was occluded at $60 \%$ and beyond and was increasing in size as the level of LAD occlusion increased. The biplane view shown in Fig. 8(b) indicates the presence of ischemia in the apical region even when the LAD is not completely occluded.

By comparing Fig. 8(b) and (c), it is possible to observe the ischemic region growing towards the lateral, posterior, and septal walls with increasing level of LAD occlusion. The purpose of that experiment was to show that the EWI was sensitive to changes in the electrical conduction pattern as a result of disease; further studies on a larger animal sample size are needed to show that EWI can be used to diagnose mild or intermediate ischemia. Currently, the echocardiographic stress test may allow detection of early ischemia. However, such tests are limited by low image quality caused by fast heart rates, potential contrast administration requirements, and the limited time available to acquire an image. Moreover, for patients who cannot perform the stress test, drugs such as dobutamine may need to be systemically administered. Therefore, EWI at rest could become a valuable complementary or alternative method to the echocardiographic stress test for early ischemic onset detection.

\section{Significance and Limitations}

The previous section showed a good agreement between the electrical and EWI patterns in the myocardium. However, the EMW is a close but not an exact representation of the electrical activation patterns. The electrical activation of a myocyte will generate, milliseconds later, contraction along the myofibers. If we considered this effect alone, the EMW pattern would then be expected to follow and be a delayed version of the electrical activation pattern. However, the heart is subject to additional stresses and strains. The mechanical activation in one location may generate strains in the neighboring tissue and throughout the myocardium. Other phenomena involving the hemodynamics of the heart or the valve function will also modify the expected strain patterns. Consequently, the electromechanical delay, that is, the delay between the electrical activation and the mechanical activation is not expected to be uniform throughout the myocardium in the normal case and changes with the electrical activation sequence [38]. The transmural variation of the electromechanical delay in vitro [39] and in vivo [31] has also been documented and showed a spatial variation of the electromechanical delay across the ventricle. The electromechanical delay might also vary with the definition of the mechanical activation used. For example, a definition of the mechanical activation similar to the definition of the EMW but using circumferential strains obtained using MR-based methods was reported in [20], [40], and showed good agreement between the mechanical and electrical activation sequences when pacing from the right-ventricular wall, hence corroborating the results presented here. Several studies using implanted markers, e.g., sonomicrometry [41] or biplane cineradiography [42], have described the sequence of circumferential and longitudinal mechanical activation during sinus rhythm [43]-[45] and related it to the electrical activation [41]. Those studies, however, tended to show that the mechanical activation on the epicardium and endocardium follows an apex-to-base propagation pattern in the lateral wall. The complex EMW propagation patterns obtained with EWI emphasizes that a high spatial and temporal resolution are needed to obtain an accurate depiction of the mechanics of the heart during the QRS complex of the sinus rhythm. EWI allowed, for the first time, to our knowledge, the observation of the cardiac excitation-contraction coupling in full echocardiographic views in vivo with high spatial and temporal resolution using a noninvasive, noncontact methodology. For example, we have been able to isolate temporally and spatially the strains resulting from the electrical activation from the strains generated after the mitral valve closure. 
A limitation of the current implementation of EWI, however, is that in the regions where the ultrasound beam is not clearly aligned to the cardiac radial coordinates, the interpretation of the incremental strains becomes more complex, because, in such cases, the assumption that axial direction matches, or has the largest projection component in, the radial direction may not entirely hold. The general case is that the axial direction will be a linear combination of the radial and circumferential or longitudinal directions. Longitudinal shortening and radial thickening onsets may not occur simultaneously in general [41], hence the position of the probe might have an influence on the activation times patterns obtained in specific regions of the heart such as the apical region of the lateral wall. A solution to the angle-dependence of the method would be to estimate the displacements and strains in 2-D. Such estimations can be more sensitive to noise and, despite being beyond the scope of this paper, constitute the object of ongoing studies [8], [46]. On the other hand, even if the regions where the angle dependence might be important, the EMW propagation pattern in a specific echocardiographic view could constitute a diagnostic tool in itself, as it maps directly the excitation-contraction coupling throughout the myocardium. Despite the relatively small sample size, the fact that the EMW consistently shows similar patterns of propagation in the canine heart suggests that it could constitute a useful tool for the noninvasive assessment of the cardiac function, e.g., it could be used to measure the synchrony of ventricular contraction or the ability of the myocardial tissue to undergo mechanical activation.

\section{Conclusion}

This paper introduced new methods, parameters, and applications for electromechanical wave imaging (EWI). The motion-matching technique allowed the reconstruction of a fullview ciné-loop without the use of ECG. By imaging the incremental strains associated with the EMW, we defined the onset of the EMW and generated isochronal maps along multiple echocardiographic planes. Those maps were similar to the electrical activation maps previously reported in the literature. Finally, EWI was found to be sensitive to intermediate ischemia, detecting regions at $60 \%$ coronary occlusion and beyond. Those results indicate that EWI could be used in the future to assess electrical conduction properties of the myocardium, and detect ischemic onset and disease progression both entirely noninvasively.

\section{Acknowledgments}

This work was supported in part by the National Institutes of Health under Grant R01EB006042 and in part by the Wallace H. Coulter Foundation. The work of J. Provost was supported in part by the Natural Sciences and Engineering Research Council of Canada (NSERC) and in part by Fonds Québécois de la Recherche sur la Nature et les Technologies (FQRNT).

The authors wish to thank J. Wang for conducting open-heart surgery; E. Ciaccio, E. Hyodo, A. Danpinid, A. Safarov, and I. Sebro for their help during experiments; and H. S. Duffy, P. Danilo, and I. N. Shlapakova for their advice on the experimental procedure. The authors also wish to thank J. Luo, V. Gurev, and N. Trayanova for helpful discussions.

\section{References}

1. Pernot M, Konofagou EE. Electromechanical imaging of the myocardium at normal and pathological states. Proc IEEE Ultrason Symp. Sep.2005 2:1091-1094.

2. Pernot M, Fujikura K, Fung-Kee-Fung SD, Konofagou EE. ECG-gated, mechanical and electromechanical wave imaging of cardiovascular tissues in vivo. Ultrasound Med Biol. 2007; 33(7):1075-1085. [PubMed: 17507146]

3. Konofagou, EE.; Luo, J.; Saluja, D.; Fujikura, K.; Cervantes, D.; Coromilas, J. Noninvasive electromechanical wave imaging and conduction velocity estimation in vivo. Proc. IEEE Ultrason. Symp; Oct. 2007; p. 969-972. 
4. Bers DM. Cardiac excitation-contraction coupling. Nature. 2002; 415(6868):198-205. [PubMed: 11805843]

5. Li Y, Garson CD, Xu Y, Beyers RJ, Epstein FH, French BA, Hossack JA. Quantification and MRI validation of regional contractile dysfunction in mice post myocardial infarction using high resolution ultrasound. Ultrasound Med Biol. 2007; 33(6):894-904. [PubMed: 17434660]

6. Sutherland GR, Salvo GD, Claus P, D'hooge J, Bijnens B. Strain and strain rate imaging: A new clinical approach to quantifying regional myocardial function. J Am Soc Echocardiogr. 2004; 17(7): 788-802. [PubMed: 15220909]

7. Konofagou EE, D’hooge J, Ophir J. Myocardial elastography-a feasibility study in vivo. Ultrasound Med Biol. 2002; 28(4):475-482. [PubMed: 12049961]

8. Lee W, Ingrassia C, Fung-Kee-Fung S, Costa K, Holmes J, Konofagou E. Theoretical quality assessment of myocardial elastography with in vivo validation. IEEE Trans Ultrason, Ferroelect, Freq Control. Nov; 2007 54(11):2233-2245.

9. Luo J, Fujikura K, Homma S, Konofagou EE. Myocardial elastography at both high temporal and spatial resolution for the detection of infarcts. Ultrasound Med Biol. 2007; 33(8):1206-1223. [PubMed: 17570577]

10. Chen X, Xie H, Erkamp R, Kim K, Jia C, Rubin JM, O’Donnell M. 3-D correlation-based speckle tracking. Ultrason Imag. 2005; 27(1):21-36.

11. Wang S, Lee Wn, Provost J, Luo J, Konofagou EE. A composite high-frame-rate system for clinical cardiovascular imaging. IEEE Trans Ultrason, Ferroelect, Freq Control. Oct; 2008 55(10): 2221-2233.

12. Kanai H, Satoh H, Hirose K, Chubachi N. A new method for measuring small local vibrations in the heart using ultrasound. IEEE Trans Biomed Eng. Dec; 1993 40(12):1233-1242. [PubMed: 8125499]

13. Provost, J.; Gurev, V.; Trayanova, N.; Konofagou, EE. Characterization of wave origins in electromechanical wave imaging. presented at the IEEE Int. Ultrasonics Symp; Beijing, China. Nov. 2008;

14. Malkin RA, Kramer N, Schnitz B, Gopalakrishnan M, Curry AL. Advances in electrical and mechanical cardiac mapping. Physiol Meas. 2005; 26(1):R1-R14. [PubMed: 15742872]

15. Chattipakorn N, Fotuhi PC, Chattipakorn SC, Ideker RE. Three-dimensional mapping of earliest activation after near-threshold ventricular defibrillation shocks. J Cardiovas Electrophysiol. 2003; 14(1):65-71.

16. Chattipakorn N, KenKnight BH, Rogers JM, Walker RG, Walcott GP, Rollins DL, Smith WM, Ideker RE. Locally propagated activation immediately after internal defibrillation. Circulation. 1998; 97(14):1401-1410. [PubMed: 9577952]

17. Schilling RJ, Peters NS, Davies DW. Simultaneous endocardial mapping in the human left ventricle using a noncontact catheter: Comparison of contact and reconstructed electrograms during sinus rhythm. Circulation. 1998; 98(9):887-898. [PubMed: 9738644]

18. Yue AM, Paisey JR, Robinson S, Betts TR, Roberts PR, Morgan JM. Determination of human ventricular repolarization by noncontact mapping: Validation with monophasic action potential recordings. Circulation. 2004; 110(11):1343-1350. [PubMed: 15353505]

19. Zhang X, Ramachandra I, Liu Z, Muneer B, Pogwizd SM, He B. Noninvasive three-dimensional electrocardiographic imaging of ventricular activation sequence. Am J Physiol Heart Circulatory Physiol. 2005; 289(6):H2724-2732.

20. Faris OP, Evans FJ, Ennis DB, Helm PA, Taylor JL, Chesnick AS, Guttman MA, Ozturk C, Mcveigh ER. Novel technique for cardiac electromechanical mapping with magnetic resonance imaging tagging and an epicardial electrode sock. Ann Biomed Eng. 2003; 31(4):430-440. [PubMed: 12723684]

21. Kallel F, Ophir J. A least-squares strain estimator for elastography. Ultrason Imag. 1997; 19(3): 195-208.

22. Lai, WM.; Rubin, D.; Krempl, E. Introduction to Continuum Mechanics. 3. New York: Pergamon; 1993. 
23. Luo J, Konofagou EE. High-frame rate, full-view myocardial elastography with automated contour tracking in murine left ventricles in vivo. IEEE Trans Ultrason, Ferroelect, Freq Control. Jan; 2008 55(1):240-248.

24. Cspedes I, Huang Y, Ophir J, Spratt S. Methods for estimation of subsample time delays of digitized echo signals. Ultrason Imag. 1995; 17(2):142-171.

25. Cerqueira MD, Weissman NJ, Dilsizian V, Jacobs AK, Kaul S, Laskey WK, Pennell DJ, Rumberger JA, Ryan T, Verani MS. Standardized myocardial segmentation and nomenclature for tomographic imaging of the heart: A statement for healthcare professionals from the cardiac imaging committee of the council on clinical cardiology of the American heart association. J Am Soc Echocardiogr. 2002; 15(5):463-467.

26. Liu Z, Kastis GA, Stevenson GD, Barrett HH, Furenlid LR, Kupinski MA, Patton DD, Wilson DW. Quantitative analysis of acute myocardial infarct in rat hearts with ischemia-reperfusion using a high-resolution stationary SPECT system. J Nucl Med. 2002; 43(7):933-939. [PubMed: 12097466]

27. Farb A, Kolodgie F, Jenkins M, Virmani R. Myocardial infarct extension during reperfusion after coronary artery occlusion: Pathologic evidence. J Am College Cardiol. 1993; 21(5):1245-1253.

28. Scher AM, Young AC. The pathway of ventricular depolarization in the dog. Circul Res. 1956; 4(4):461-469.

29. Durrer D, Dam RTV, Freud GE, Janse MJ, Meijler FL, Arzbaecher RC. Total excitation of the isolated human heart. Circulation. 1970; 41(6):899-912. [PubMed: 5482907]

30. Sengupta PP, Tondato F, Khandheria BK, Belohlavek M, Jahangir A. Electromechanical activation sequence in normal heart. Heart Fail Clin. 2008; 4(3):303-314. [PubMed: 18598982]

31. Ashikaga H, Coppola BA, Hopenfeld B, Leifer ES, McVeigh ER, Omens JH. Transmural dispersion of myofiber mechanics: Implications for electrical heterogeneity in vivo. J Am College Cardiol. 2007; 49(8):909-916.

32. Stylen A, Slrdahl S, Skjelvan GK, Heimdal A, Skjaerpe T. Strain rate imaging in normal and reduced diastolic function: Comparison with pulsed doppler tissue imaging of the mitral annulus. J Am Soc Echocardiogr. 2001; 14(4):264-274. [PubMed: 11287889]

33. Sengupta PP, Khandheria BK, Korinek J, Jahangir A, Yoshifuku S, Milosevic I, Belohlavek M. Left ventricular isovolumic flow sequence during sinus and paced rhythms: New insights from use of high-resolution doppler and ultrasonic digital particle imaging velocimetry. J Am College Cardiol. 2007; 49(8):899-908.

34. Remme EW, Lyseggen E, Helle-Valle T, Opdahl A, Pettersen E, Vartdal T, Ragnarsson A, Ljosland M, Ihlen H, Edvardsen T, Smiseth OA. Mechanisms of preejection and postejection velocity spikes in left ventricular myocardium: Interaction between wall deformation and valve events. Circulation. 2008; 118(4):373-380. [PubMed: 18606917]

35. Holmes JW, Borg TK, Covell JW. Structure and mechanics of healing myocardial infarcts. Ann Rev Biomed Eng. 2005; 7:223-253. [PubMed: 16004571]

36. Villarreal FJ, Lew WY, Waldman LK, Covell JW. Transmural myocardial deformation in the ischemic canine left ventricle. Circul Res. 1991; 68(2):368-381.

37. Vatner SF. Correlation between acute reductions in myocardial blood flow and function in conscious dogs. Circul Res. 1980; 47(2):201-207.

38. Gurev V, Constantino J, Trayanova NA. Transmural dyssynchrony of myofiber shortening is determined by depolarization sequence within myocardial layers. Circulation. 2008; 118(18):S349-350.

39. Cordeiro JM, Greene L, Heilmann C, Antzelevitch D, Antzelevitch C. Transmural heterogeneity of calcium activity and mechanical function in the canine left ventricle. Am J Physiol Heart Circ Physiol. 2004; 286(4):H1471-1479. [PubMed: 14670817]

40. McVeigh E, Faris O, Ennis D, Helm P, Evans F. Electromechanical mapping with MRI tagging and epicardial sock electrodes. J Electrocardiol. 2002; 35:61-64. [PubMed: 12539100]

41. Sengupta PP, Khandheria BK, Korinek J, Wang J, Jahangir A, Seward JB, Belohlavek M. Apex-toBase dispersion in regional timing of left ventricular shortening and lengthening. J Am College Cardiol. 2006; 47(1):163-172. 
42. Ashikaga H, van der Spoel TI, Coppola BA, Omens JH. Transmural myocardial mechanics during isovolumic contraction. JACC: Cardiovasc Imag. 2009; 2(2):202-211.

43. Kirn B, Starc V. Continuous axial contraction wave in the free wall of the guinea pig left ventricle. Comput Biol Med. 2007; 37(10):1394-1397. [PubMed: 17161833]

44. Kirn B, Starc V. Contraction wave in axial direction in free wall of guinea pig left ventricle. Am J Physiol Heart Circ Physiol. 2004; 287(2):H755-759. [PubMed: 15059778]

45. Iribe G, Ward CW, Camelliti P, Bollensdorff C, Mason F, Burton RA, Garny A, Morphew MK, Hoenger A, Lederer WJ, Kohl P. Axial stretch of rat single ventricular cardiomyocytes causes an acute and transient increase in ca2+ spark rate. Circ Res. 2009; 104(6):787-795. [PubMed: 19197074]

46. Luo J, Konofagou EE. Effects of various parameters on lateral displacement estimation in ultrasound elastography. Ultrasound Med Biol. 2009; 35(8):1352-1366. [PubMed: 19525061] 

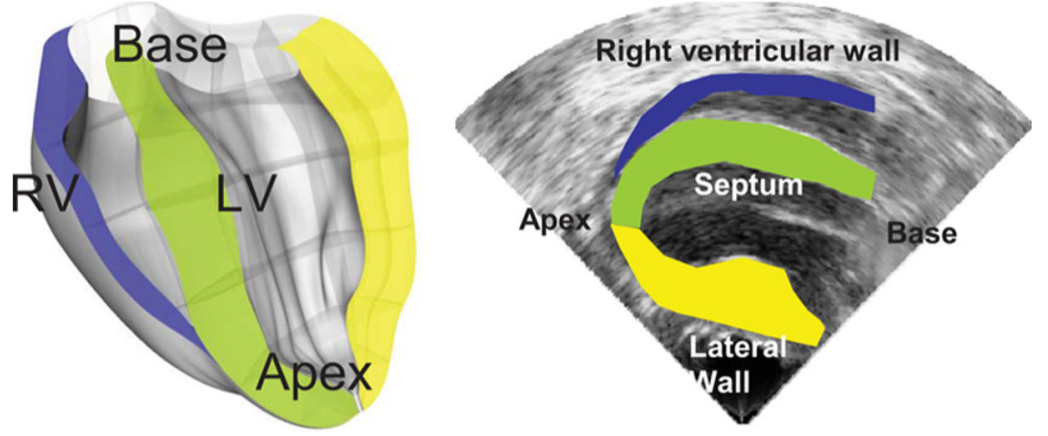

(a)
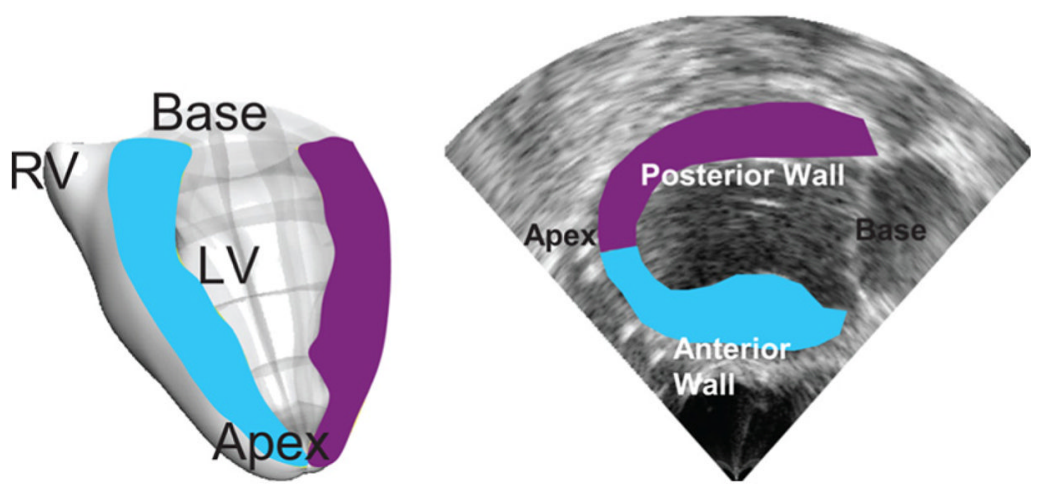

(b)

Fig. 1.

(a) Parasternal four-chamber view. Both the right (RV) and left ventricular (LV) cavities are visible, along with the septum (green), the lateral wall (yellow) and the right ventricular wall (purple). (b) Parasternal two-chamber view. The left-ventricle cavity is visible, along with the posterior and anterior walls. In both views, the axial direction coincided with the cardiac radial direction in the majority of the myocardium imaged. 


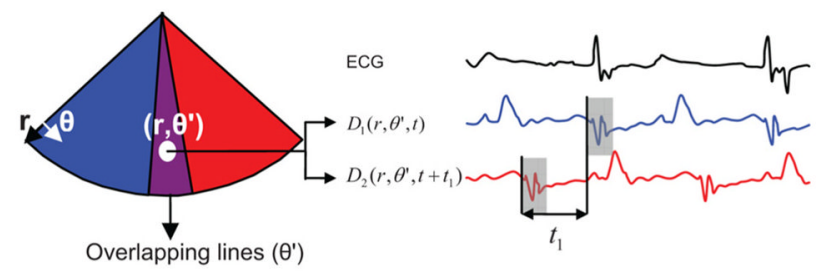

(a)

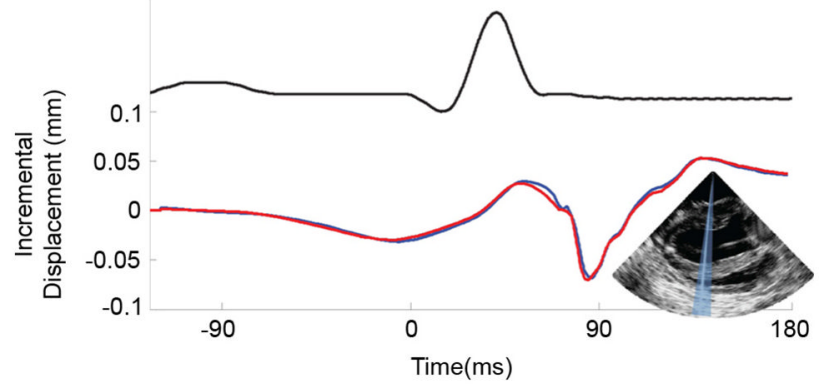

(b)

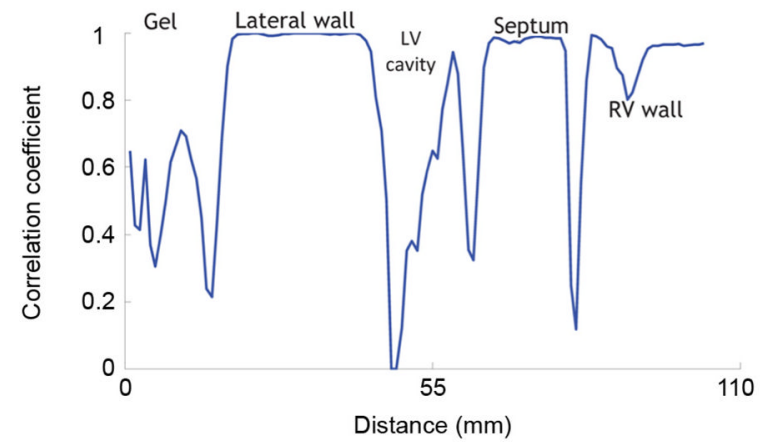

(c)

Fig. 2.

Illustration of the motion-matching method. (a) Axial incremental displacement is estimated twice in the overlapping RF-beams: once when sector 1 is acquired, and once when sector 2 is acquired. Assuming the periodicity of the heart, cross-correlation between those two independent acquisitions provides the time delay between the two sectors. (b) Incremental axial displacement over time of a sample located in the lateral wall acquired from an overlapping RF-beam. The blue line was acquired with sector 1 and the red line, with sector 2. One can observe the similarity between the two curves and the experimental periodicity of the heart's incremental displacements. (c) Cross-correlation coefficients of the incremental axial displacement over time along an overlapping RF-beam. These coefficients are close to 1 in the myocardial tissue, and dramatically drop in the cavity and in the ultrasound gel. 


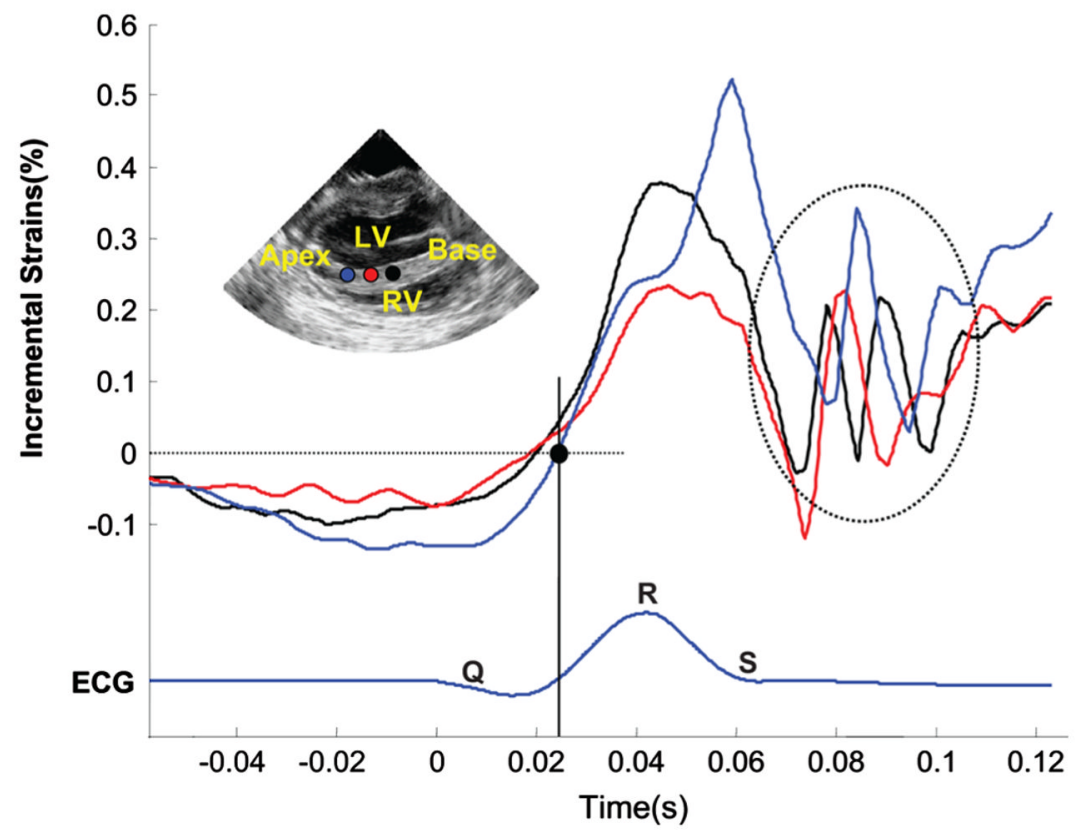

Fig. 3.

Temporal variation of the incremental strains along three points in the septum in the vicinity of the QRS complex. The electromechanical wave (EMW) is defined by the point at which the incremental strains change sign. The EMW is initiated at the endocardial surface of the septum (red) and travels towards the base (black) and the apex (blue). Oscillations (dashed circle) are also observed following the mitral valve closure. These travel from base (black) to apex (blue). 

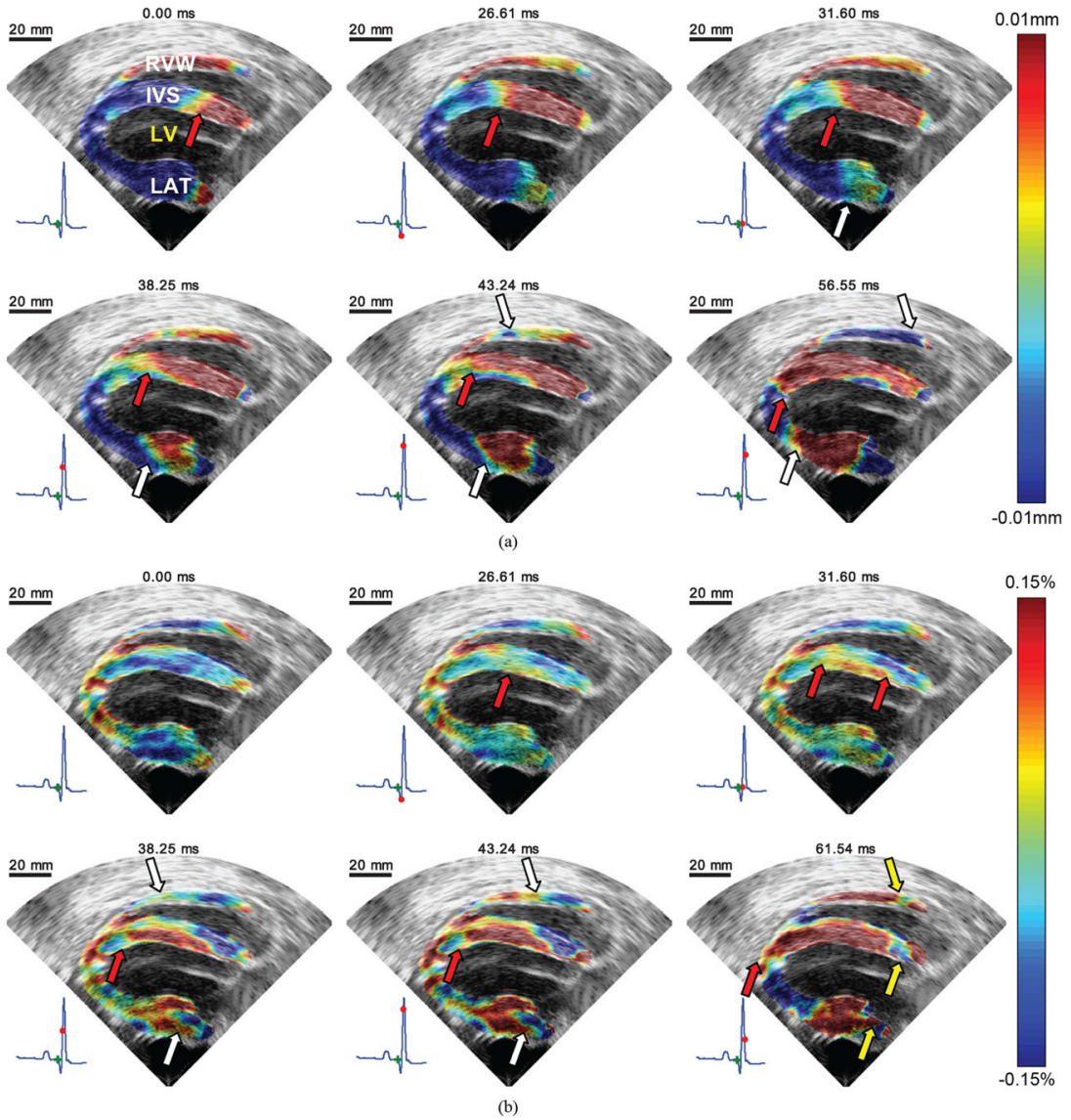

Fig. 4.

EWI during sinus rhythm in the parasternal four-chamber view in the normal ventricles. RVW, IVS, LV, and LAT, respectively, denote right ventricle wall, interventricular septum, left ventricle cavity and free wall (lateral wall). (a) Incremental displacement images. Displacements away and towards the transducer are depicted in red and blue, respectively. A wave traveling from base to apex is clearly visible in the septum. (b) Incremental strain images. Approximately $30 \mathrm{~ms}$ after the onset of the QRS, excitation of the upper endocardium of the left ventricle begins. It then propagates both towards the base and the apex (red arrows). Mechanical activation also appears in the lateral wall and travels mostly towards the base. $40 \mathrm{~ms}$ after the onset of the QRS, the right ventricular wall begins to thicken. $60 \mathrm{~ms}$ after the onset of the QRS, the myocardium is still not completely mechanically activated; thinning of portions of the base are still visible (yellow arrows). At $61.54 \mathrm{~ms}$, negative incremental strains are observed in the apical region. This is most likely due to the fact that in this region, the axial direction contains a large projection of the longitudinal strain component. 


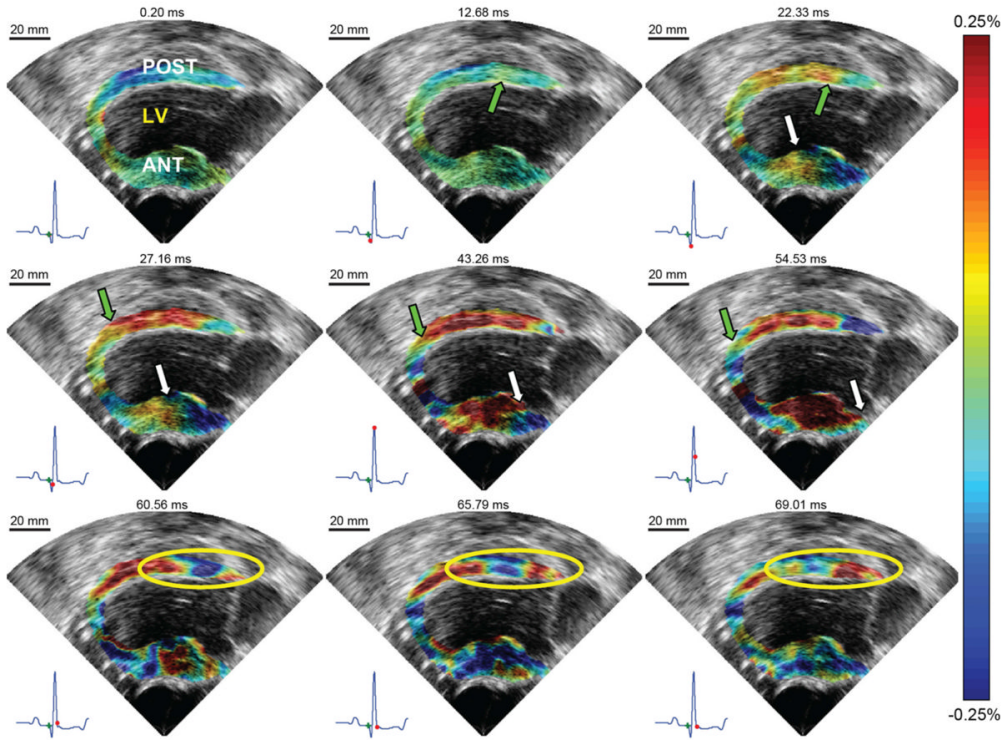

Fig. 5.

EWI of the normal heart under sinus rhythm using incremental strains in the parasternal two-chamber view. LV, ANT, and POST, respectively, denote left ventricle cavity, anterior wall, and posterior wall. Top row: Approximately $12 \mathrm{~ms}$ after the onset of the QRS, excitation of the posterior wall endocardium begins. It then propagates both towards the base and the apex. A few milliseconds later, the anterior wall begins to thicken. Middle row: $45 \mathrm{~ms}$ after the onset of the QRS, the myocardium is almost completely mechanically activated; thinning of portions of the base are still visible. Bottom row: After the mitral valve closes, an oscillating wave (yellow circle), different in nature from the EMW, is observed. It emanates from the base and travels towards the apex. 


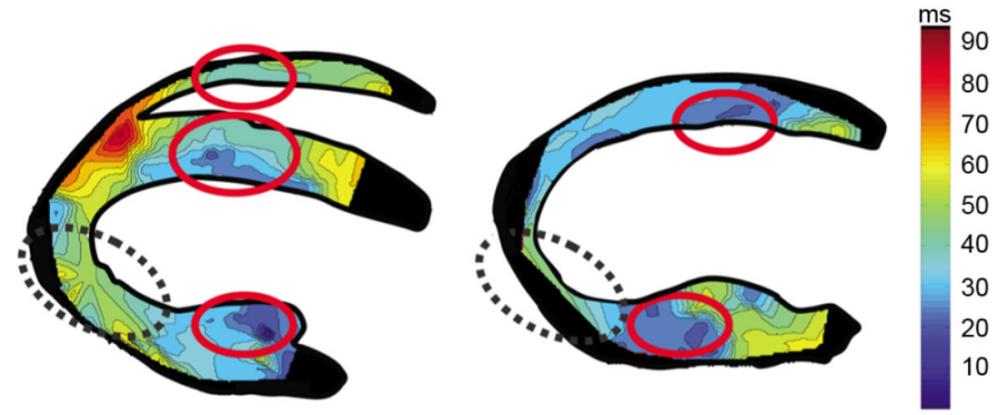

(a)
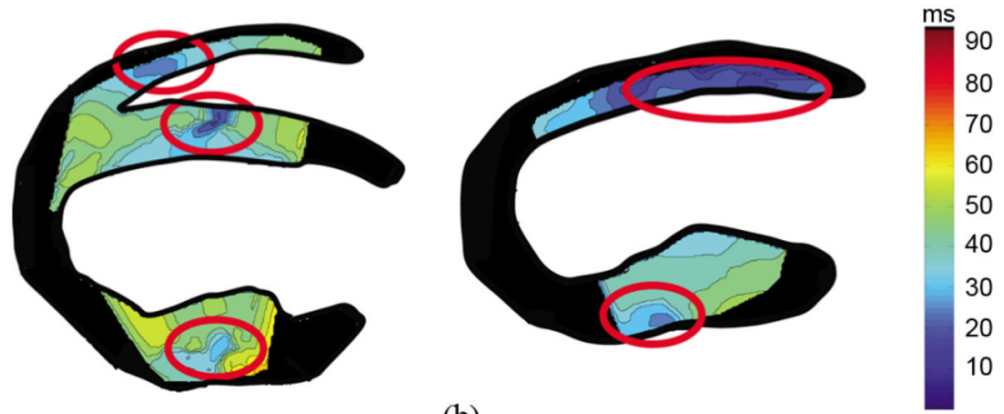

(b)

Fig. 6.

Isochrones in two different canine hearts (a), (b) in the four-chamber (left panel) and twochamber (right panel) views showing the time of arrival of the EMW. Early activated zones are depicted in dark blue, in $10 \mathrm{~ms}$ increments. Despite the fact that the two propagation patterns are not identical, they show similar regions of early depolarization and a propagation emanating from those regions to apex and base. The dashed circles indicate the regions where the activation time correspond to shortening rather than thickening. Regions of the myocardium for which a clear zero-crossing point could not be obtained were depicted in black. 


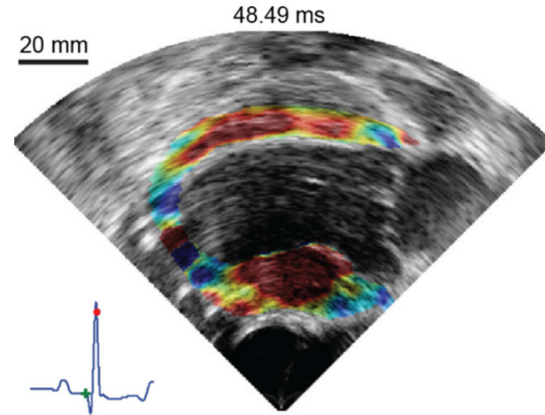

(a)

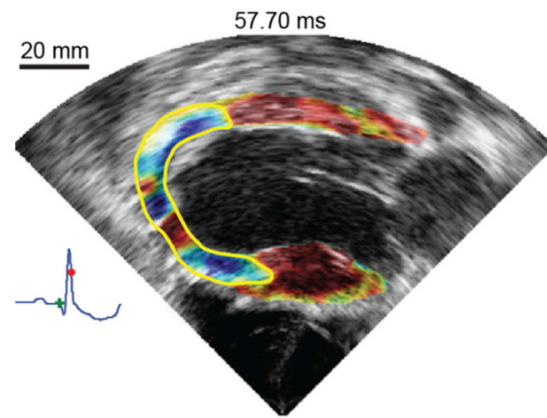

(d)

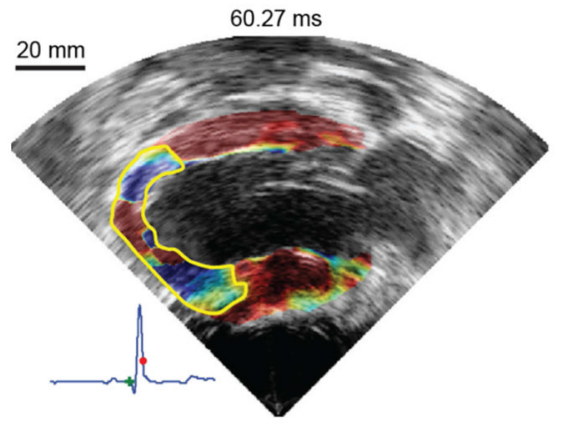

(g)

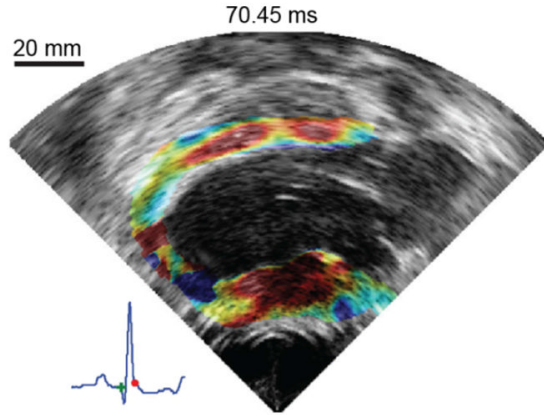

(b)

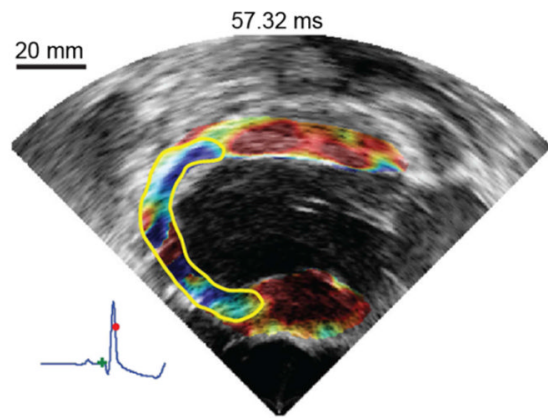

(e)

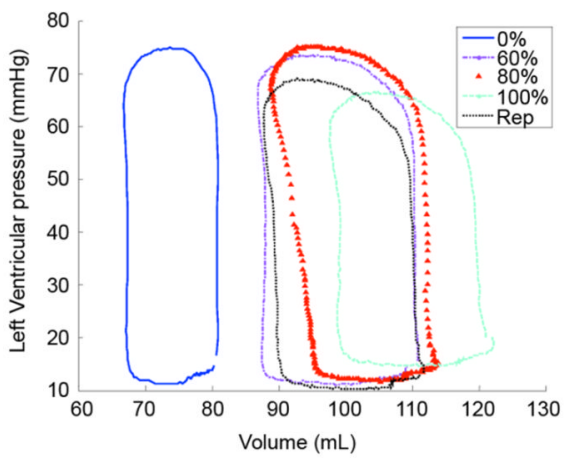

(h)

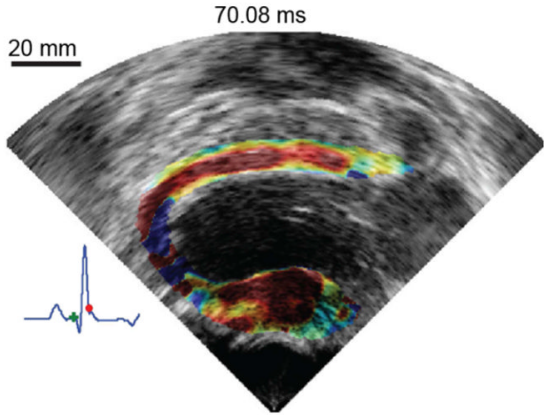

(c)

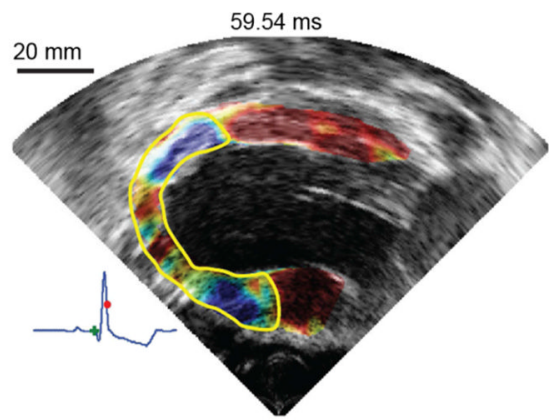

(f)

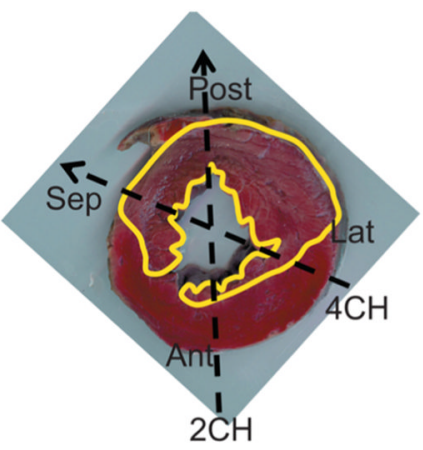

(i)

Fig. 7.

Evolution of the EMW as the occlusion level of the LAD is increasing. The time point chosen to display the EMW corresponds to the time at which the largest portion of the myocardium is activated. No significant differences were observed between (a) no occlusion and (b) $20 \%$ and (c) $40 \%$ occlusion. The ischemic region (delimited by the yellow line) becomes visible at (d) $60 \%$ occlusion and increases in size as the level of occlusion reaches (e) $80 \%$ and (f) $100 \%$. (g) After reperfusion, the ischemic region was smaller. (h) Evolution of the PV loop with the occlusion level. (i) TTC staining after infarction (reperfusion). This mid-apical slice shows the extent of the infarcted region (white) over the viable tissue (red). The parasternal two- and four-chamber views are also depicted. 

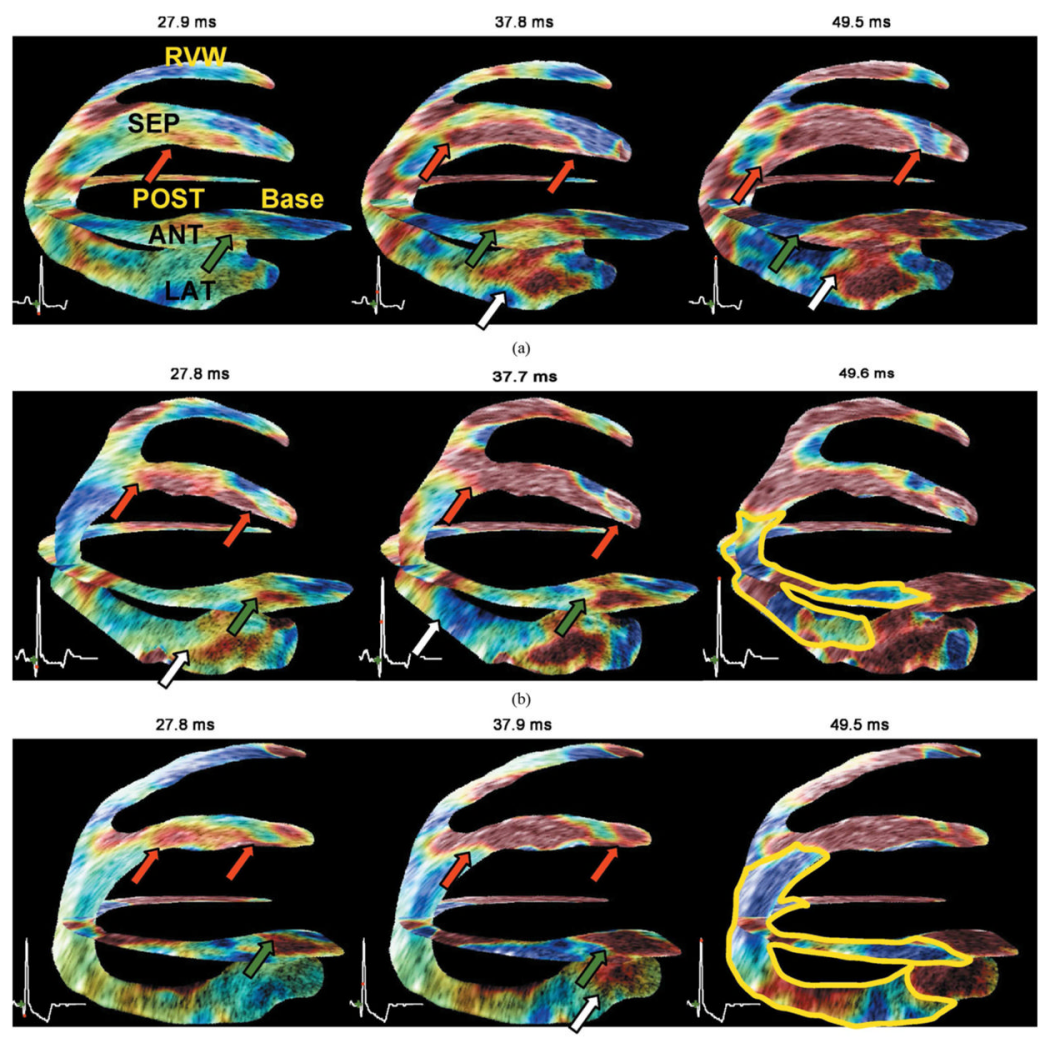

(c)

Fig. 8.

Bi-plane (two-chamber and four-chamber views) view of the same heart under different LAD coronary artery occlusion levels. Red, white, and green arrows indicate the propagation of the EMW in the septal, anterior, and lateral wall, respectively. (a) Without any occlusion, i.e., normal coronary flow, radial thinning is visible up to regions where the axial direction of the ultrasound beam coincides with the longitudinal direction of the cardiac geometry, where shortening is expected (in blue). (b) At $60 \%$ occlusion, this behavior is reversed in presence of ischemia: a region (delimited with a yellow line) containing radial thinning (blue) and longitudinal lengthening (red) is observed. (c) At complete occlusion this region increases in size. The ischemia is visible in the anterior, posterior and lateral wall near the apex at $60 \%$ occlusion and in the anterior, posterior, lateral, and septal wall at $100 \%$ occlusion. 